\title{
Overwhelming infection in asplenic patients: current best practice preventive measures are not being followed
}

\author{
D J Waghorn
}

\begin{abstract}
Aims-Patients without spleens are at increased risk of overwhelming infection. Recently, greater efforts, including the publication of national guidelines, have been made to improve the management of asplenic individuals. In theory, risks of serious sepsis can be reduced by good advice, immunisation, and antibiotic prophylaxis. In practice, such preventive measures might not be followed or may fail. A study of recent cases of overwhelming postsplenectomy infection (OPSI) was undertaken to examine specific associated factors and to determine whether currently recommended preventive measures are being followed.
\end{abstract}

Methods-Cases of OPSI were identified and reported mainly by microbiologists across the country using a specifically designed proforma. Data including the nature of the infection and vaccination/ antibiotic prophylaxis history since splenectomy were obtained.

Results-Seventy seven cases were reported. The age range varied from 3 months (congenital asplenia) to 87 years. In those who had undergone surgical splenectomy, the time interval between surgery and OPSI varied from 24 days to 65 years. Overall mortality reached $50 \%$, with underlying haematological malignancy associated with the highest death rate. Streptococcus pneumoniae caused approximately $90 \%$ episodes. Only $31 \%$ individuals had received pneumococcal vaccination before OPSI. Seven of 17 pneumococcal infections in immunised cases could be considered vaccine failures. Few patients had been adequately advised on antibiotic prophylaxis or other measures. Conclusions-Currently accepted best practice for managing asplenic patients is not being followed. Some OPSI cases may still be preventable but many asplenic individuals remain unrecognised. The compilation of asplenic patient registers might help to implement agreed policies with audit necessary to evaluate compliance. More is needed to ensure optimal management for this cohort of the population. (F Clin Pathol 2001;54:214-218)

Keywords: splenectomy; immunisation; overwhelming postsplenectomy infection; asplenic patient register

The importance of the spleen in the body's immune response to various infections and the potential consequences of its removal have become increasingly recognised over the past 40-50 years. ${ }^{1}$ Asplenic individuals can have major difficulties in coping with specific infections where the spleen would normally play a prominent role in protection. The spleen, in particular, is able to filter encapsulated bacteria such as Streptococcus pneumoniae once bloodstream invasion has occurred. ${ }^{2}$ Its absence results in an increased risk of serious sepsis carrying considerable mortality. The overall incidence of septicaemia remains low but death rates from overwhelming postsplenectomy infection (OPSI) have been reported to be up to 600 times greater than in the general population, with an estimated lifetime risk for OPSI of approximately $5 \%$. $^{3}$

The past decade has seen increased efforts to highlight the risks of infection in asplenic patients, to improve general awareness, and to give advice on appropriate precautions to prevent OPSI. Publications regarding this area have included leading journal editorials, chief medical officer newsletters, specific general practitioner mailshots, and more general media coverage. In 1996, guidelines were published describing the appropriate management for prevention and treatment of infection both in asplenic and hyposplenic individuals. ${ }^{4}$

Despite all such efforts, reports of OPSI cases continue to occur. ${ }^{5}$ A surveillance study was therefore undertaken to analyse OPSI episodes occurring across the country. The aims of our study were first to determine whether affected patients had received current best practice preventive measures and second to use the data for the further education of the relevant groups associated with the management of asplenic individuals.

\section{Methods}

In August 1994, a questionnaire style proforma was designed and copies were distributed to all members of the Association of Medical Microbiologists (AMM). This requested data on cases of OPSI occurring within their districts. It was felt that clinical microbiologists were the single most likely group of specialists to become aware of OPSI episodes and the AMM membership includes 550 microbiologists from almost all districts across the country. To alert other potential case sources, such as infectious disease and public health physicians, notices regarding the study were also published by the Public Health Laboratory Service for England and Wales ${ }^{6}$ and the Scottish Centre for Infection and Environmental Health. ${ }^{7}$ 


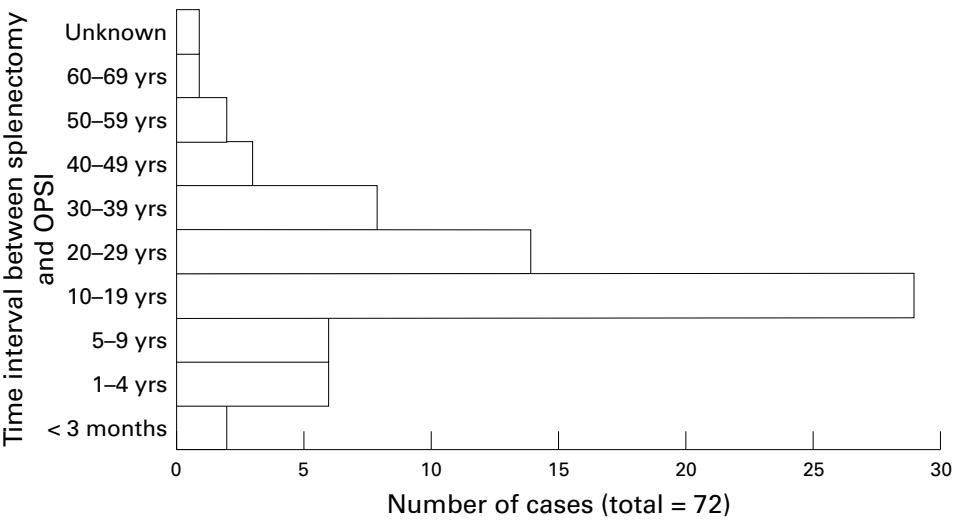

Figure 1 Time interval between splenectomy and sepsis in 72 cases of overwhelming postsplenectomy infection (OPSI).

Table 1 Underlying cause of splenectomy in 72 individuals who developed subsequent OPSI

\begin{tabular}{ll}
\hline Cause of splenectomy & $\begin{array}{l}\text { No. of cases } \\
(n=72)\end{array}$ \\
\hline Trauma & 24 \\
Haematological malignancies (including Hodgkin's lymphoma, NHL, leukaemias) & 13 \\
Other haematological disorders (including ITP, haemolytic anaemias) & 24 \\
Accidental damage at surgery & 7 \\
Other causes/unknown & 4 \\
\hline
\end{tabular}

ITP, idiopathic thrombocytopenic purpura; NHL, non-Hodgkin's lymphoma; OPSI, overwhelming postsplenectomy infection.

OPSI had been defined as septicaemia and/or meningitis, usually fulminant but not necessarily fatal, occurring at any time after removal of the spleen. The proforma requested information on the acute OPSI episode, patients' clinical background relating to their asplenic condition, any previous OPSI, and details regarding immunisation/antibiotic prophylaxis history. The carriage of a medical alert card, bracelet, etc was also to be noted. AMM members were asked to complete and return a proforma on each OPSI episode they became aware of in their district.

\section{Results}

By the end of 1998, data had been received on 77 cases. Most reported episodes occurred within the first three years of the study but there was wide geographical representation with cases reported from all areas of the UK. Overwhelming infections occurred in 48 males and 29 females with an age range from three months to 87 years. Almost half the cases occurred in patients aged 30-49. Five individuals had not undergone surgical splenectomy. These comprised three congenitally asplenic children and two adults found to have severe splenic atrophy at necropsy after their fatal sepsis. In the remaining 72 cases, the interval from splenectomy to OPSI varied from 24 days to 65 years (fig 1 ).

Haematological disease had been responsible for approximately half of the splenectomies, with malignancy involved in one third, and more benign disorders, such as haemolytic anaemia and idiopathic thrombocytopenic purpura (ITP), responsible for the remainder. One third of the cases had followed trauma (table 1). Overall mortality reached $50 \%$ but variation occurred between underlying causative groups (table 2). The highest mortality was
Table 2 Associated mortality with underlying cause of asplenia in 77 reported cases

\begin{tabular}{lll}
\hline & Survived & Died \\
\hline Total cases & $40 / 77(52 \%)$ & $37 / 77(48 \%)$ \\
Trauma cases & $13 / 24(54 \%)$ & $11 / 24(46 \%)$ \\
Haematological (total) cases & $17 / 37(46 \%)$ & $20 / 37(54 \%)$ \\
$\quad \begin{array}{l}\text { Haematological malignancy } \\
\text { Haematological other }\end{array}$ & $4 / 13(31 \%)$ & $9 / 13(69 \%)$ \\
$\quad$ condition & $13 / 24(54 \%)$ & $11 / 24(46 \%)$ \\
All other cases & $10 / 16(62 \%)$ & $6 / 16(38 \%)$ \\
\hline
\end{tabular}

Table 3 Pathogens responsible for overwhelming postsplenectomy infection episode

\begin{tabular}{ll}
\hline Pathogens & No. of cases (total $=77)$ \\
\hline Streptococcus pneumoniae & 67 \\
Neisseria meningitidis & 2 \\
Listeria monocytogenes & 1 \\
Escherichia coli & 1 \\
Klebsiella sp. & 1 \\
Salmonella typhimurium & 1 \\
Unknown & 4 \\
\hline
\end{tabular}

associated with haematological malignancy, whereas the death rate in other haematological conditions was comparable with all other causes.

By far the most common pathogen reported was $S$ pneumoniae with a small range of other organisms responsible for the remaining cases (table 3). Serotyping results on $S$ pneumoniae isolates were recorded on 49 of 67 strains, with $75 \%$ belonging to serotypes covered in the current 23 valent pneumococcal polysaccharide vaccine.

Excluding the five patients who had not undergone splenectomy, immunisation records were expected for 72 individuals. In two cases, no records were available. For the remaining 70 assessable patients, 22 had received pneumococcal vaccine before suffering their OPSI episode; postimmunisation pneumococcal antibody values had been measured in one patient. Of these 22 individuals, $S$ pneumoniae was responsible for 17 infections. Seven of the 17 could be considered possible vaccine failures (table 4), whereas in the other 10 cases, either the causative strain belonged to a serotype not present in the vaccine or the last immunisation had been given more than 10 years earlier. In the remaining 48 eligible cases not given pneumococcal vaccine, 43 infections were caused by $S$ pneumoniae. Two patients had evidently been offered but refused immunisations in the past and for one patient, who subsequently suffered a fatal OPSI, the causative pneumococcal strain would have been covered by the vaccine. In 13 individuals, pneumococcal vaccine was administered after their OPSI episode.

Regarding the other two recommended immunisations, nine and seven patients had received Haemophilus influenzae and Neisseria meningitidis vaccines, respectively, before OPSI. No case of $H$ influenzae and two cases of $N$ meningitidis infection were reported. These last two infections both occurred in patients who had received meningococcal immunisation but neither causative strain belonged to serotypes present in the current meningococcal polysaccharide vaccine and therefore were not considered as vaccine failures. 
Table 4 Details of seven OPSI cases caused by Streptococcus pneumoniae, which might represent possible vaccine failure

\begin{tabular}{|c|c|c|c|c|c|c|}
\hline Case & Cause of splenectomy & $\begin{array}{l}\text { Age at time of } \\
\text { OPSI }\end{array}$ & $\begin{array}{l}\text { Time interval between } \\
\text { vaccination and OPSI }\end{array}$ & $\begin{array}{l}\text { Pneumococcal } \\
\text { serotype }\end{array}$ & $\begin{array}{l}\text { Patient taking } \\
\text { antibiotic } \\
\text { prophylaxis at } \\
\text { time of OPSI }\end{array}$ & Fatal episode \\
\hline 17 & Accidental damage at surgery & 47 & 5 years & $12 \mathrm{~F}$ & No & Yes \\
\hline 22 & Hodgkin's disease & 32 & 15 months & $22 \mathrm{~F}$ & Yes* & Yes \\
\hline 33 & Haemolytic anaemia & 33 & 2 months & $23 \mathrm{~F}$ & No & No \\
\hline 35 & Non-Hodgkins lymphoma & 52 & 2 years & $6 \mathrm{~B}$ & Yest & No \\
\hline 53 & Idiopathic thrombocytopenic purpura & 33 & 8 months & $9 \mathrm{~V}$ & No & No \\
\hline 67 & Accidental damage at surgery & 63 & 18 months & $7 \mathrm{~F}$ & No & No \\
\hline 77 & Unspecified lymphoproliferative haematological disorder & 35 & 4 years & 20 & No & Yes \\
\hline
\end{tabular}

${ }^{\star}$ On penicillin $250 \mathrm{mg}$ twice daily, with infecting pneumococcal strain sensitive to penicillin.

†On erythromycin $500 \mathrm{mg}$ daily, with infecting pneumococcal strain resistant to erythromycin.

OPSI, overwhelming postsplectomy infection.

As with immunisation records, an antibiotic prophylaxis history of some description was expected in the 72 asplenic individuals who had undergone surgery. Data revealed that antibiotics had been taken for variable periods between splenectomy and OPSI by 17 patients, with 10 of 17 (14\% total cases) taking prophylaxis at the time of infection. In the remaining 62 cases not taking regular prophylaxis, two had been prescribed antibiotics to keep at home for self medication at early signs of possible infection. One patient had been issued with a medical alert card detailing his asplenic condition.

\section{Discussion}

Although the data from this OPSI surveillance study highlight important and continuing areas of concern in the management of asplenic individuals, it is acknowledged that this series of reported cases will not have represented all OPSI episodes occurring in the country during the study period. However, our study is likely to have reflected the overall picture and clearly shows that life threatening sepsis persists despite the recent increased efforts to improve professional management and public education on the condition. There has been no reduction in mortality, with a death rate of $50 \%$, rising to $70 \%$ in underlying haematological malignancies; similar figures have been described before. Another disturbing statistic is that almost two thirds of cases occurred in those under 50 years of age, many of whom were in otherwise good health with no additional risk factors for fulminant infection.

An important message can be drawn from the data on the time interval between splenectomy and OPSI episode. Previous reports have consistently stated that the highest OPSI risk lies within the first few years after surgery. ${ }^{8}$ Our analysis demonstrates that the increased risk of severe sepsis is lifelong. Almost the same number of cases occurred more than 40 years after surgery as were seen within the first four years of surgery, with most cases $(60 \%)$ occurring 10-30 years after splenectomy. The risk of life threatening sepsis varies with the reason for splenectomy and any underlying illness. ${ }^{4}$ Our study confirms that associated malignancy, especially haematological, carried the highest risk, but all asplenic patients should receive optimal advice and protection whatever the underlying aetiology.

The most dangerous pathogen for asplenic individuals is unquestionably $S$ pneumoniae. At least $87 \%$ episodes were caused by this organism, yet only $31 \%$ patients had received pneumococcal vaccine before OPSI. This is important when considering that $75 \%$ of infecting strains belonged to serotypes present in the current 23 valent polysaccharide vaccine. Immunisation has become accepted as an integral part of the preventive strategy against serious infection in the asplenic population, ${ }^{9}$ but OPSI episodes classified as vaccine failures are regularly described. ${ }^{10}$ Indeed, under use of the pneumococcal vaccine has been attributed to controversy about its effectiveness in high risk patients. ${ }^{11}$ Few controlled studies have demonstrated vaccine efficacy in asplenic patients but two recent reports support its use. (1) A programme of pneumococcal vaccination and prophylactic antibiotics effectively prevented serious infection in a population of 280 children for an average of 4.3 years after splenectomy ${ }^{12}$; and (2) in a series of over 200 adult asplenic haematology patients who had been immunised, only four episodes of pneumococcal sepsis were seen in 13 years and in all four episodes the infecting serotype was not included in the vaccine. ${ }^{13}$

The appearance of possible vaccine failure cases was anticipated in this series. Underlying severe haematological disease usually associated with immunosuppression was present in three of seven episodes. Therefore, the response to immunisation might have been poor, yet no attempt had been made to measure antibody responses to vaccination in these patients, and overall only one individual who had received pneumococcal vaccine had undergone this test. Some patients with so called vaccine failure might have benefited from antibody values being checked and, where appropriate, booster immunisations offered.

There are difficulties with antibody responses in asplenic patients, especially in relation to the pneumococcal polysaccharide vaccine. Up to $20 \%$ of asplenic individuals may show a poor immunological response, with antibody values declining more rapidly than in those still possessing a spleen. ${ }^{14}$ Second, in contrast to the accepted protective antibody value for $H$ influenzae type $b$, there is no agreed protective titre against $S$ pneumoniae, and the current measurement of pneumococcal antibodies gives a total titre against the 23 valent polysaccharide vaccine, whereby a good response against one serotype might mask a failed or poor response to other serotypes. 
Decisions on the timing of booster immunisations are also difficult but the proposal to base this on antibody measurement has been made. The optimal frequency for checking antibody titres has yet to be established but two recommendations have been published. The first suggested antibody measurement preimmunisation, one month after, and then at three and five years postimmunisation, ${ }^{15}$ whereas the second group advocated a three week postimmunisation check then measurement at yearly intervals. ${ }^{16}$ Individuals who fail to respond could be targeted for other measures such as maintaining lifelong antibiotic prophylaxis.

In this series, despite the lack of an OPSI caused by $H$ influenzae and only two episodes caused by $N$ meningitidis, both organisms have been reported as important potential pathogens in asplenic patients, ${ }^{17}$ and the advice to immunise against them should be supported. In recent years, much research has taken place to improve the immunogenicity of pneumococcal and meningococcal vaccines in particular. This has already resulted in the introduction of a new meningococcal conjugate type vaccine although protection is confined to serogroup C strains. It is hoped that similar advances in immunisation against serogroup B meningococci and pneumococcal pathogens will follow.

The use of antibiotic prophylaxis remains controversial because there is a lack of good clinical data demonstrating its efficacy in asplenic patients. ${ }^{18} \mathrm{~A}$ prospective nationally based study would be required to confirm benefit, but such data would be impossible to gather. Patient records in our study highlight the difficulties in assessing prophylaxis efficacy. Only 10 individuals were taking prophylaxis at the time of OPSI. In these cases, $S$ pneumoniae caused nine of 10 infections and prophylactic penicillin or amoxycillin failed to prevent sepsis in six episodes, despite the isolates being sensitive to penicillin in vitro. The specific prophylactic regimen was not reported for two patients, but in one case, prophylactic erythromycin was being taken and the infecting pneumococcal strain was resistant to the macrolide agent. Increasing antimicrobial resistance, especially to commonly used antibiotics, such as the penicillins or macrolides, has provoked concerns over continuous antibiotic prescription. This issue, together with patient compliance difficulties, has influenced some to advocate a policy of holding a reserve antibiotic supply for self prescribing at the earliest signs of infection. ${ }^{19}{ }^{20}$ If this policy is considered increasingly appropriate, the finding here that only two of 62 potential candidates possessed a reserve antibiotic course, demonstrates the need for further education of both patients and clinicians.

The advice for asplenic individuals to be issued with a form of medical alert, such as a card or a bracelet, ${ }^{521}$ has two purposes. First, it should provide a constant reminder to the individual of their underlying condition and, second, knowledge of their asplenic state might be vital for medical attendants in the event of any medical emergency. Because only one person was reported to hold a card, this aspect of patient management needs increased attention. The possibility that the outcome of a few OPSI episodes might have been affected by the potential lack of knowledge of the patient's asplenic state at presentation of sepsis cannot be excluded.

In conclusion, using data from published evidence, it is estimated that there are approximately 50000 asplenic individuals living in the UK. It is accepted that this cohort runs an increased risk of life threatening sepsis. However, the real risk has never been clarified for large populations on a long term basis. Existing published data suggest an incidence of OPSI varying from 0.18 to 0.42 cases $/ 100$ person years. ${ }^{22}{ }^{23}$ Although an expectation of preventing all OPSI is unrealistic, this study suggests a continuing broad failure to attain currently accepted best practice in the management of asplenic patients. It is probable that following recent efforts to raise awareness, there has been improved vaccine uptake and antibiotic prophylaxis counselling in those who have recently undergone splenectomy. However, individuals who lost their spleen more than five to 10 years ago are more likely to have missed out on protective measures, a conclusion supported here because only $11 \%$ of OPSI cases had occurred within four years of surgery. Optimal care must be given to all asplenic patients but the first step requires their detection. Three groups have published results on the foundation of asplenic patient registers at district or regional level. ${ }^{162425}$ The close involvement of primary care, hospital clinicians, public health departments, and the patients themselves is crucial for the success of such programmes, both to discover previously unrecognised cases and to contribute to the immunisations/antibiotic prophylaxis/general counselling required. An assessment of the patients' own knowledge about their condi$\operatorname{tion}^{26}$ can help in the formulation of educational programmes. Once agreed policies are introduced into a district or region, it is important to evaluate compliance by performing audit. ${ }^{25}$ Overall, there is still improvement needed to achieve best practice for the management of asplenic patients so that the goal of lowering the number of OPSI episodes to an irreducible minimum can be achieved.

1 Shaw JHF, Print CG. Postsplenectomy sepsis. Br f Surg 1989;76:1074-81.

2 Jenks PJ, Jones E. Infections in asplenic patients. Clin Microbiol Infect 1996;1:266-72.

3 Lynch AM, Kapila R. Overwhelming postsplenectomy infection. Infect Dis Clin North Am 1996;10:693-707.

4 Working Party of the British Committee for Standards in Haematology Clinical Haematology Task Force. Guidelines for the prevention and treatment of infection in patients with an absent or dysfunctional spleen. BMF 1996;312:430-4.

5 Ong ELC, Hassan ISA, Snow MH. Pneumococcal sepsis in a splenectomised patient. Br f Gen Pract 1995;45:502-3.

6 Anonymous. Surveillance of overwhelming infection following splenectomy. Public Health Lab Service CDR Weekly 1994;4:169.

7 Anonymous. Overwhelming infection following splenecSCIEH Weekly Report 1995;29:1.

8 Fielding AK. Prophylaxis against late infection following splenectomy and bone marrow transplant. Blood Rev 1994;

9 Long SG, Smith AG, Perry BA, et al. Implementing a policy for pneumococcal prophylaxis in a haematology unit after splenectomy. Qual Health Care 1995;4:194-6. 
10 Klinge J, Hammersen G, Scharf J, et al. Overwhelming postsplenectomy infection with vaccine-type Streptococcus pneumoniae in a 12-year-old girl despite vaccination and antibiotic prophylaxis. Infection $1997 ; 25: 368-71$.

11 Shann F. Modern vaccines: pneumococcus and influenza. Lancet 1990;335:898-901.

12 Konradsen HB, Henrichsen J. Pneumococcal infections in splenectomised children are preventable. Acta Paediatr Scand 1991;80:423-7.

13 Abildgaard N, Nielsen JL. Pneumococcal septicaemia and meningitis in vaccinated splenectomised adult patients. Scand $\mathcal{F}$ Infect Dis 1994;26:615-17.

14 Hazlewood M, Kumararatne DS. The spleen. Who needs it anyway? Clin Exp Immunol 1992;89:327-9.

15 Kassianos GC. Uncertainty exists over frequency of blood tests to test antipneumococcal immunity. BMF 1996;312: 1360-1.

16 Spickett GP, Bullimore J, Wallis J, et al. Northern region asplenia register - analysis of first two years. F Clin Pathol asplenia register

17 Spelman DW. Postsplenectomy overwhelming sepsis: reducing the risks. Med $\mathscr{f}$ Aust 1996;164:648.

18 Read RC, Finch RG. Prophylaxis after splenectomy. $f$ Antimicrob Chemother 1994;33:4-6.
19 Finch RG, Read R. Lifelong penicillin may be ineffective. BMF 1994:308:132.

20 Waghorn DJ. Prevention of postsplenectomy sepsis. Lancet 1993;341:248

21 Mayon-White R. Protection for the asplenic patient. Prescribers Fournal 1994;34:165-70.

2 Schwartz PE, Sterioff S, Mucha P, et al. Postsplenectomy sepsis and mortality in adults. $\mathcal{F} A M A$ 1982;248:2279-83.

23 Cullingford GL, Watkins DN, Watts ADJ, et al. Severe late postsplenectomy infection. Br f Surg 1991;78:716-21.

24 Sarangi J, Coleby M, Trivella M, et al. Prevention of post splenectomy sepsis: a population based approach. F Public Health Med 1997;19:208-12.

25 MacInnes J, Waghorn DJ, Haworth E. Management of asplenic patients in south Buckinghamshire: an audit of local practice. Commun Dis Rep CDR Rev 1995:5:R173-7.

26 Waghorn DJ, Haworth E. Avoiding infection after splenectomy. Br f Gen Pract 1996;46:691.

27 Rasmussen C, Eystrud P, Hansen JB, et al. Asplenic patients' knowledge of prophylactic measures against patients knowledge of prophylactic measur.

28 Kind EA, Craft C, Fowles JB, et al. Pneumococcal vaccine administration associated with splenectomy: missed opportunities. Am F Infect Control 1998;26:418-22.

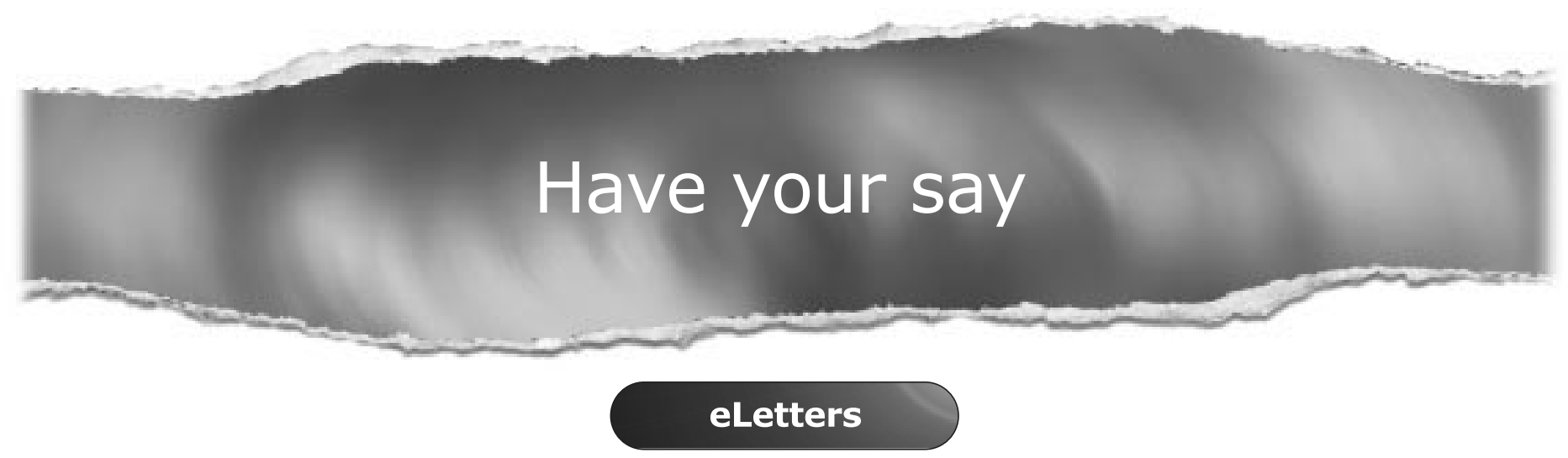

If you wish to comment on any article published in the Journal of Clinical Pathology you can send an eLetter using the eLetters link at the beginning of each article. Your response will be posted on Journal of Clinical Pathology online within a few days of receipt (subject to editorial screening).

\section{www.jclinpath.com}

